\title{
Patienteninformation \\ und Krankenhausreputation
}

\author{
Andrea Eichmüller, Thomas Helbling, Sabina Heuss, Susan Göldi
}

alle FHNW Fachhochschule Nordwestschweiz, Hochschule für Wirtschaft

Die Digitalisierung macht auch vor Patientinnen und Patienten nicht halt. 2017 zählt das Internet zu den meistgenutzten Informationsquellen bei der Recherche zu Gesundheitsthemen. Bei der Krankenhauswahl sind nach wie vor die Empfehlungen der Zuweisenden, die Reputation und Mundpropaganda entscheidend, wobei sich diese zunehmend in die digitale Welt verlagern.

Wissenschaftliche Untersuchungen bestätigen die Erfahrungen der Ärzteschaft: Das Internet hat auch in der Informationssuche rund um Gesundheitsthemen weltweit eine deutliche Relevanz - Tendenz steigend. Im Jahr 2000 suchten 25\% der US-Amerikanerinnen und -Amerikaner mit Internetanschluss online nach Gesundheitsinformationen. 2012 waren es bereits $72 \%$. In Europa zeichnet sich ein ähnliches Bild [1]. Selbst die Schweiz kann sich dieser Entwicklung nicht entziehen. Gemäss einer Studie von Swisscom haben 2011 84\% der Schweizerinnen und Schweizer bereits einmal gesundheitsbezogene Informationen im Internet recherchiert. Lediglich $12 \%$ gaben an, das Internet noch nie für die Recherche von gesundheitsbezogenen Informationen genutzt zu haben [2].

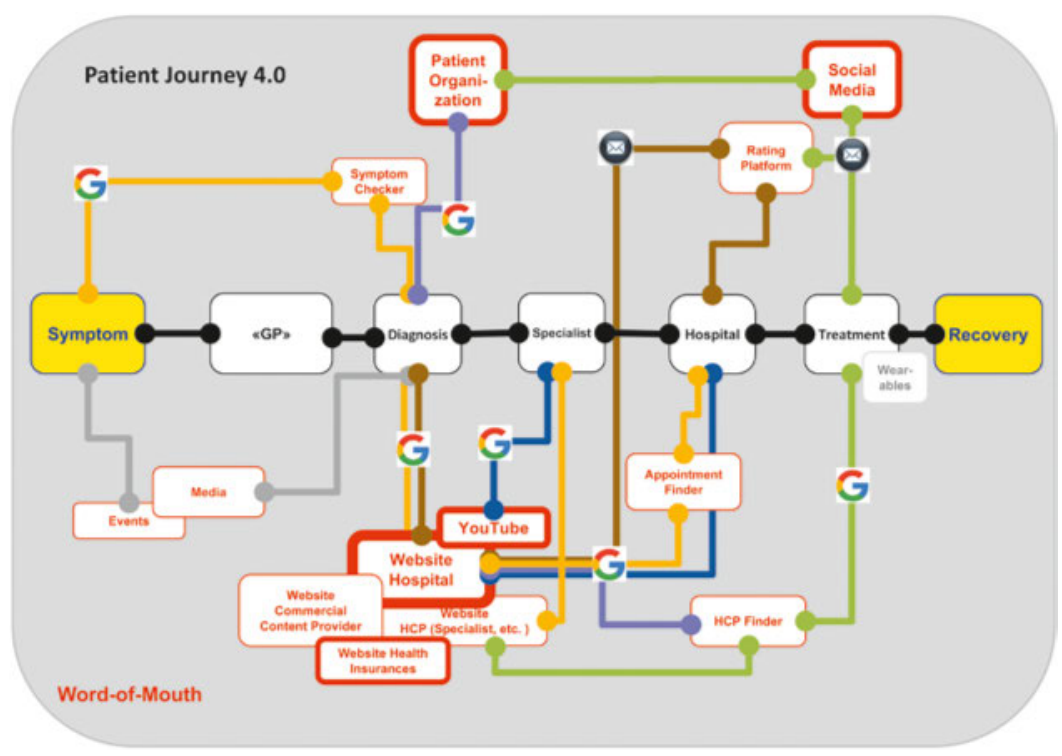

Abbildung 1: Patient Journey im digitalen Zeitalter (eigene Darstellung)
Auch der Swiss eHealth Barometer 2017 zeigt, dass klassische Medien als Informationsquellen bei Gesundheitsfragen zwar nach wie vor relevant sind, jedoch zunehmend an Bedeutung verlieren. Das Internet ist auf dem Vormarsch. 2017 nutzten so viele Befragte das Internet respektive Gesundheitsportale oder Apps wie nie zuvor. Dies dürfte sich in den kommenden Jahren noch verstärken, da die Wahl des Mediums zur Informationsbeschaffung in Gesundheitsfragen klar von Generationeneffekten geprägt ist. Personen $65+$ nutzen web- und mobilebasierte Kanäle weniger. Umgekehrt verhält es sich hingegen bei den nachfolgenden Altersgruppen, die stark auf Online-Kanäle setzen [3].

\section{Informationsquelle Dr. Google}

Gemäss Google recherchieren 77\% der Amerikanerinnen und Amerikaner online, bevor sie einen Arzttermin vereinbaren [4]. In der Schweiz sind es $84 \%$, die sich vor oder nach einem Arzttermin online über Symptome und Krankheiten informieren [5]. Dies ist wenig überraschend, ist das Phänomen «Dr. Google» doch allseits bekannt und die Selbstdiagnose oftmals nicht fern. 35\% der Amerikanerinnen und Amerikaner stellen sich oder anderen online eine Diagnose [6]. Dabei starten weltweit die meisten Diagnosen mit der Eingabe in einer Suchmaschine wie Google, Bing oder Yahoo [7, 8]. Weitere zentrale Informationsquellen sind Webseiten von Krankenhäusern (76\%, wobei $43 \%$ über Suchmaschinen den Weg auf die Krankenhauswebseite finden) sowie gesundheitsspezifische Informationsseiten (52\%) [9]. In der Schweiz verhält sich dies nicht anders: 82\% der Schweizerinnen und Schweizer nutzen Suchmaschinen als Einstiegsseite, um sich 
über gesundheitsbezogene Themen zu informieren, gefolgt von Gesundheitsportalen (58\%), Wikipedia (49\%) und Diskussionsforen (36\%) [10]. Das Themenspektrum, zu dem im Internet recherchiert wird, ist breit, wie eine amerikanische Studie 2013 aufzeigt: In über $70 \%$ der Fälle suchen die medizinischen Laien nach Informationen zu gesundheitlichen Problemen unterschiedlicher Schwere und nach Beiträgen zu medizinischen Erfahrungen oder Problemen anderer (26\%). Weitere 20\% suchen online nach Rezensionen und Rankings von Arzneimitteln, medizinischen Behandlungen, Ärzten oder Krankenhäusern sowie nach Personen, mit denen sie ihre gesundheitlichen Sorgen teilen können (16\%) [11]. Besonders beliebt sind dabei Patienten-Testimonials, d.h. von Leidensgenossen erstellte Erfahrungsberichte [12]. Dies ist nicht weiter erstaunlich. Mundpropaganda, ob persönlich oder online, wird als vertrauenswürdigste Informationsquelle erachtet, was in besonderem Masse für das Gesundheitswesen gilt [13].

\section{Krankenhauswahl im Zeitalter der Digitalen Revolution}

Aus Daten einer englischen und einer amerikanischen Studie wissen wir einiges darüber, worauf Patientinnen und Patienten einen Entscheid für ein Krankenhaus stützen. Die Daten harmonieren mit Erfahrungen im Markt der schweizerischen Krankenhäuser und scheinen deshalb übertragbar. Knapp 65\% der Patientinnen und Patienten sind in die Wahl des Krankenhauses involviert. 41\% treffen die Entscheidung zusammen mit ihrem Arzt und 24\% entscheiden in erster Linie für sich alleine oder mit ihrer Familie [14]. Auch hier lassen sich Generationeneffekte erkennen: Patientinnen und Patienten der Altersgruppe 70+ folgen strikt dem Rat ihres Arztes; Patientinnen und Patienten der Altersgruppe 51-69 nehmen zwar den Rat ihrer Ärztin an, recherchieren ihre Optionen aber online; Patientinnen und Patienten der Altersgruppe 35-50 suchen aktiv online nach Informationen. Sie orientieren sich an Gesundheitsmarken und konsultieren oftmals soziale Netzwerke, bevor sie eine Entscheidung fällen. Für Patientinnen und Patienten der Altersgruppe 18-34 ist wiederum die Patientenerfahrung entscheidend [15].

Die Konsultation von Dr. Google ist aber nicht gleichzusetzen mit der Wahl des Krankenhauses. Marshall und McLoughlin [16] gehen in ihrer englischen Studie 2010 davon aus, dass der Entscheidungsprozess zur Krankenhauswahl kein rationaler Prozess, sondern ein sozialer, iterativer ist. Die Wahl des Krankenhauses wird oft von der Grösse, Verfügbarkeit und Distanz der
Institution abhängig gemacht - dazu werden die persönliche Einstellung und die eigene momentane Stimmung und Verfassung in den Entscheidungsprozess mit einbezogen. Diese Aussagen aus England werden gestützt durch deutsche Studien. Zum Beispiel aus den Jahren 2009 und 2011 [17, 18]. Trotz genügend Vorbereitungszeit und damit ausreichend Zeit für eine gute Recherche im Internet (es handelt sich in dieser Studie um Patientinnen und Patienten vor einem elektiven operativen Eingriff), wird schlussendlich auf Grund der Vorerfahrung mit dem Krankenhaus, der Wohnortnähe und der Qualifikation des Arztes entschieden. Im Vordergrund stehen ausserdem die persönliche Information und der Aufbau von Vertrauen durch ambulante Vorkontakte. Die Studien zeigen, dass Familie, Freunde und Bekannte (noch) die wichtigere Entscheidungshilfe zu sein scheinen als das Internet. Dieses hingegen wird vor allem für die Diagnose und das Therapieverfahren, sowie für Informationen über das Krankenhaus genutzt. Zur Wahl des Krankenhauses selbst wird es nicht herbeigezogen und stellt nur eine erste Orientierungshilfe dar oder wird für weiterführende Informationen zu Rate gezogen.

\section{Patientenerfahrung startet online}

Schlagworte wie der «informierte Patient» und «shared decision making» sind die Grundlagen des modernen Arzt-Patienten-Verhältnisses. Einfach verfügbare Informationen aus dem Internet können, je nach Medienkompetenz der Patientinnen und Patienten (Stichwort: health literacy), zu einer verbesserten Zusammenarbeit zwischen Patient und Ärztin führen. Das Nachrücken der «Digital Natives» dürfte zu einer Verschiebung der Einflussgrössen auf die Krankenhauswahl führen. Die Bedeutung der Reputation und der Mundpropaganda dürften dabei unverändert bleiben oder gar zunehmen. Daraus ergeben sich zwei Grundsätze für die Online-Präsenz von Krankenhäuser:

\section{Die Bedeutung der Online-Reputation wird zunehmen}

Unbestritten ist, dass jüngere Patientinnen und Patienten sowie deren Angehörige und betreuende Personen zunehmend im Internet nach Informationen suchen. Noch werden diese Informationen nicht zur Wahl des Krankenhauses benutzt, sondern vor dem Krankenhausaufenthalt und besonders danach als Informationsquelle und Kommentarmöglichkeit herbeigezogen. Vorausgegangene Erfahrungen und Markenerlebnisse im Zusammenhang mit einem Krankenhaus werden auch in Zukunft wichtig bleiben und zur Wahl des Krankenhauses beitragen. Dann aber nicht nur off- 
line, sondern vermehrt auch online stattfinden. Die On- und Offline-Reputation eines Krankenhauses wird demnach an Wichtigkeit zulegen.

Um die Reputation zu steigern, braucht es eine eindeutige Positionierung im Markt und damit einhergehend einen unverkennbaren Auftritt des Krankenhauses gegen innen und aussen. Nur so kann das wichtigste Gut eines Krankenhauses, das Vertrauen der Patientinnen und Patienten, gewonnen und erhalten werden. Das medizinische Fachpersonal, Ärztinnen und Ärzte, Pflegefachkräfte und medizinisch-technische Expertinnen und Experten fungieren dabei als Quelle für vertrauenswürdige und "gesicherte» medizinische Informationen. Krankenhäuser sollten diese machtvolle Quelle an Qualitätsinformationen nutzen und das Feld nicht selbsternannten Medizinexpertinnen und -experten überlassen. Krankenhäuser, die sich nicht aktiv um ihre Online-Präsenz kümmern, geben künftig ihre Reputation in die Hände der Community.

\section{Erfahrungen werden in Zukunft häufiger online geteilt werden}

Bereits heute teilen viele Patientinnen und Patienten ihre Erfahrungen nach einem Arzttermin. 50\% sprechen mit der Familie, Freundinnen und Kollegen, 18\% verfassen einen Beitrag in sozialen Netzwerken oder auf Webseiten, zeigt eine grosse amerikanische Studie [19]. In der Schweiz wird das kaum wesentlich anders sein. Denn Entscheidungen beruhen generell vor allem auf eigenen Erfahrungen sowie jenen des sozialen Umfeldes. Dieses Umfeld breitet sich durch das Internet aber aus: Was früher Familie, Freunde und Bekannte waren, ist bereits heute durch Austauschund Bewertungsplattformen beträchtlich erweitert worden. Kommentare und Erfahrungen von zwar unbekannten, aber als kompetent und authentisch wahrgenommenen Personen tragen zunehmend als erweitertes soziales Umfeld zur Entscheidungsfindung bei. In der Schweiz ist unter vielen anderen comparis mit einer Bewertungsplattform aktiv. $\mathrm{H}+$ bietet einen Spitalbarometer. Krankenhäuser sollten sich aktiv in die öffentliche Diskussion einbringen und die Meinungsfindung im Web nicht anderen überlassen. Das Engagement auf den relevanten Plattformen sollte ebenso Aufgabe der Kommunikationsabteilung wie der Kliniken sein. Krankenhäuser werden in Zukunft die Wahl haben, ob potentielle Patientinnen und Patienten mit ihnen sprechen oder Informationen aus dem Internet holen. Über sie wird im Netz auf jeden Fall gesprochen.

\section{Bildnachweis}

FHNW

\section{Literatur}

1 IMS Institute of Healthcare Informatics (2014), http://www. imshealth.com/files/web/IMSH\%2OInstitute/Reports/Engaging\%2OPatients\%2OThrough\%20Social\%2OMedia/IIHI_Social_ Media_Report_2014.pdf

2 Swisscom Studie (2011), https://www.swisscom.ch/dam/swisscom/ de/connected-health/documents/Studienresultate_Swisscom_ Health.pdf

3 Swiss eHealth Barometer 2017: Öffentliche Meinung, Studie im Auftrag von InfoSocietyDays, März 2017, https://www.infosocietydays.ch/index.php?apid=503931\&apparentid=5979492

4 Google / Compete Hospital Study (2012), https://ssl.gstatic.com/ think/docs/the-digital-journey-to-wellness-hospital-selection research-studies.pdf

5 Swisscom Studie (2011), https://www.swisscom.ch/dam/swisscom/ de/connected-health/documents/Studienresultate_Swisscom Health.pdf

6 Pew Research Center (2013), http://www.pewinternet.org/2013/01/ 15/health-online-2013/

7 Pew Research Center (2013), http://www.pewinternet.org/2013/01/ 15/health-online-2013/

8 Google / Compete Hospital Study (2012), https://ssl.gstatic.com/ think/docs/the-digital-journey-to-wellness-hospital-selection research-studies.pdf

9 Google / Compete Hospital Study (2012), https://ssl.gstatic.com/ think/docs/the-digital-journey-to-wellness-hospital-selection research-studies.pdf

10 Swisscom Studie (2011), https://www.swisscom.ch/dam/swisscom/ de/connected-health/documents/Studienresultate_Swisscom Health.pdf

11 Pew Research Center (2013), http://www.pewinternet.org/2013/01/ 15/health-online-2013/

12 Google / Compete Hospital Study (2012), https://ssl.gstatic.com/ think/docs/the-digital-journey-to-wellness-hospital-selection research-studies.pdf

13 Google / Compete Hospital Study (2012), https://ssl.gstatic.com/ think/docs/the-digital-journey-to-wellness-hospital-selection research-studies.pdf

14 BMJ, Information in Practice (2005), http://www.bmj.com/content/ bmj/331/7520/821.full.pdf

15 Marketing Health Services (2015), https://www.ama.org/ publications/MarketingHealthServices/Pages/the-age-factor.aspx

16 Marshall M, McLoughlin V. (2010). How do patients use information on health providers? BMJ 2010;341:c5272

17 de Cruppé W, Geraedts M. «Wie wählen Patienten ein Krankenhaus für elektive operative Eingriffe?» BundesgesundheitsblattGesundheitsforschung-Gesundheitsschutz 54.8 (2011): 951.

18 Geuter G, Weber J. Informationsbedarf chronisch kranker Menschen bei der Krankenhauswahl: Untersucht unter besonderer Berücksichtigung des Internets. Diss. Inst. für Pflegewissenschaft, 2009.

19 Google / Compete Hospital Study (2012), https://ssl.gstatic.com/ think/docs/the-digital-journey-to-wellness-hospital-selection research-studies.pdf 